\title{
EQUIVARIANT LOCALIZATION
}

\section{J. P. MAY, J. McCLURE AND G. TRIANTAFILLOU}

The purpose of this note is to construct and characterize localizations of $G$-spaces, where $G$ is a compact Lie group. It was written at the request of Reinhard Schultz, who needed its results in work of his own. When $G$ is finite, the possibility of such a construction is folklore familiar to the experts. That the general case presents little more difficulty comes as something of a surprise. Of course, restriction to integral primes is essential to such a space level, unstable, construction. One cannot expect, for example, to localize general $G$-spaces (as opposed to infinite loop $G$-spaces) at prime ideals of the Burnside ring.

We are very grateful to Terry Wall for his careful reading of both this paper and its sequel on equivariant completion.

We shall work in the category of based $G$-spaces (with $G$-fixed basepoint). Write $\pi(X, Y)_{G}$ for the set of $G$-homotopy classes of based $G$-maps $X \rightarrow Y$. A $G$-map $f: X \rightarrow Y$ is said to be a weak $G$-equivalence if its restriction $f^{H}: X^{H} \rightarrow Y^{H}$ to $H$-fixed point spaces is an ordinary weak equivalence for each closed subgroup $H$ of $G$; subgroups are understood to be closed henceforward. If $X$ and $Y$ are $G-C W$ complexes, then $f$ is necessarily a $G$-homotopy equivalence. More generally, the following result holds $[2,10,16]$.

Proposition 1. If $X$ is a G-CW complex and $f: Y \rightarrow Z$ is a weak G-equivalence, then

$$
f_{*}: \pi(X, Y)_{G} \rightarrow \pi(X, Z)_{G}
$$

is a bijection.

For any $G$-space $X$, there is a $G$-CW complex $\Gamma X$ and a weak $G$-equivalence $\alpha: \Gamma X \rightarrow X$ (see [16]). On the homotopy category $h G \mathscr{T}$ of based $G$-spaces, $\Gamma$ is a functor and $\alpha$ is natural. We define

$$
[X, Y]_{G}=\pi(\Gamma X, \Gamma Y)_{G} .
$$

By the proposition, $[X, Y]_{G} \cong \pi(X, Y)_{G}$ if $X$ is a $G$-CW complex. With the obvious composition, $[X, Y]_{G}$ gives the set of morphisms $X \rightarrow Y$ in the category $H G \mathscr{T}$ obtained from $h G \mathscr{T}$ by formally inverting the weak $G$-equivalences. This is the appropriate category in which to study localizations.

We shall need a special case of the following rough analog of Proposition 1, which seems to be new; we defer the proof to the end of the paper. 
Proposition 2. Let $E_{*}$ be any (nonequivariant) homology theory. Let $f: Y \rightarrow Z$ be a G-map such that each

$$
f_{*}^{H}: E_{*}\left(Y^{H}\right) \rightarrow E_{*}\left(Z^{H}\right)
$$

is an isomorphism. Then each

is also an isomorphism.

$$
(f / H)_{*}: E_{*}(Y / H) \rightarrow E_{*}(Z / H)
$$

In line with these results, we define homotopical properties of $G$-spaces by reference to fixed point spaces.

Definition 3. A $G$-space $X$ is said to be $G$-connected or $G$-simply connected if each $X^{H}$ is connected or simply connected. $X$ is said to be $G$-simple if each $X^{H}$ is connected and simple. $X$ is said to be $G$-nilpotent if each $X^{H}$ is connected and nilpotent and if, for each $n \geqslant 1$, the orders of nilpotency of the $\pi_{1}\left(X^{H}\right)$-groups $\pi_{n}\left(X^{H}\right)$ for varying $H$ have a common bound (a vacuous restriction if $G$ is finite).

Our basic tool will be Bredon cohomology $H_{G}^{*}(X ; M)$, as defined in [2] for finite $G$ and generalized to compact Lie groups $G$ by several authors $[6,17]$; see $[8,9]$ for the further generalization to an $\operatorname{RO}(G)$-graded cohomology theory. Here $M$ is a coefficient system, that is, a contravariant functor from the homotopy category $\mathcal{C}$ of orbit spaces $G / H$ and $G$-maps to the category of Abelian groups. We shall need the following invariance statement.

Proposition 4. Let $R$ be a commutative ring and let $M$ be an $R$-module valued coefficient system. Let $f: Y \rightarrow Z$ be a $G$-map such that each

is an isomorphism. Then

$$
f_{*}^{H}: H_{*}\left(Y^{H} ; R\right) \rightarrow H_{*}\left(Z^{H} ; R\right)
$$

$$
f^{*}: H_{G}^{*}(Z ; M) \rightarrow H_{G}^{*}(Y ; M)
$$

is an isomorphism.

Proof. There is a functorial universal coefficients spectral sequence $\left\{E_{r} X\right\}$ which converges to $H_{G}^{*}(X ; M)$ and satisfies

$$
E_{2}^{p, q} X=\operatorname{Ext}_{R c^{p}}^{p, q}\left(\mathbf{H}_{*}(X ; R), M\right),
$$

where the Ext groups are those of the Abelian category of $R$-module valued coefficient systems and where the (graded) coefficient system $\mathbf{H}_{*}(X ; R)$ is specified by

$$
\mathbf{H}_{*}(X ; R)(G / H)=H_{*}\left(X^{H} / W_{0} H ; R\right),
$$

$W_{0} H$ being the identity component of $N_{G} H / H$. See Bredon [2, I $\left.\S 10\right]$ for finite $G$ and Willson [17] in the general case (or see [9]). By hypothesis if $G$ is finite and by Proposition 2 in general, $E_{2} f$ is an isomorphism.

We shall be concerned with the case $R=Z_{T}$, where $T$ is a set of primes. We say that a coefficient system $M$ is $T$-local if each $M(G / H)$ is a $T$-local Abelian group ( $=Z_{T}$-module). Note that the localization $Z \rightarrow Z_{T}$ induces a localization

$$
M=M \otimes Z \rightarrow M \otimes Z_{T}=M_{T}
$$


of coefficient systems for any $M$. We say that a $G$-map $f: Y \rightarrow Z$ is a $T$-cohomology isomorphism if

$$
f^{*}: H_{G}^{*}(Z ; M) \rightarrow H_{G}^{*}(Y, M)
$$

is an isomorphism for all $T$-local coefficient systems $M$. The previous proposition gives a simple criterion. Of course, we also have the obvious nonequivariant notion of a $T$-cohomology isomorphism.

For a $G$-space $X$, the homotopy groups $\pi_{n}\left(X^{H}\right)$ give a coefficient system $\pi_{n} X$. An Eilenberg-MacLane $G$-space $K(M, n)$ is a $G$-CW complex such that $\pi_{n} K(M, n)=M$ and $\pi_{q} K(M, n)=0$ for $q \neq n$. A nice functorial construction of Eilenberg-MacLane $G$-spaces has been given by Elmendorf [4], and any two spaces $K(M, n)$ are $G$-homotopy equivalent. We have

$$
\tilde{H}_{G}^{n}(X ; M)=[X, K(M, n)]_{G} .
$$

If one likes, one can take this as the definition of Bredon cohomology. We use it to prove the following observation. Note that, for any $G$-spaces $X$ and $Y$, we have

$$
\left[(G / H)_{+} \wedge X, Y\right]_{G} \cong[X, Y]_{H}
$$

where $A_{+}$denotes the disjoint union of a $G$-space $A$ and a $G$-fixed basepoint. When $X=X^{H}$, the right side is $\left[X, Y^{H}\right]$.

Lemma 5. Let $G$ act trivially on a based space $X$. Then

$$
\tilde{H}_{G}^{*}\left((G / H)_{+} \wedge X ; M\right) \cong \tilde{H}^{*}(X ; M(G / H))
$$

Therefore, if $f: Y \rightarrow Z$ is a nonequivariant $T$-cohomology isomorphism, then $1 \wedge f:(G / H)_{+} \wedge Y \rightarrow(G / H)_{+} \wedge Z$ is an equivariant T-cohomology isomorphism.

Proof. This is immediate from $K(M, n)^{H}=K(M(G / H), n)$.

We consider $G$-spaces built up from spaces $K(M, n)$.

Definition 6. A $G$-tower $X$ is an inverse limit of $G$-spaces $X_{n}$ such that $X_{0}=\{*\}$ and $X_{n+1}$ is the fibre of a $G$-map $k_{n}: X_{n} \rightarrow K\left(M_{n}, q_{n}\right)$, where $q_{n} \geqslant 2$ (so that $X$ is $G$-connected). Strictly speaking, we regard the entire sequence $\left\{X_{n}\right\}$ as part of the structure of $X$. We have the following special types of $G$-towers. and

(i) A $G$-tower $X$ is simple if $q_{n}=n+2$ for $n \geqslant 0$, in which case $M_{n}=\pi_{n+1} X$

$$
k_{n}=k^{n+2} \in H_{G}^{n+2}\left(X_{n} ; \pi_{n+1} X\right)
$$

is an equivariant $k$-invariant.

(ii) A $G$-tower $X$ is nilpotent if $q_{n} \geqslant q_{m}$ for $n>m$ and only finitely many $q_{n}=i$ for each $i$; that is, the homotopy group systems $\pi_{i} X$ are built up in order, with each one built up in finitely many stages.

(iii) A $G$-tower $X$ is $T$-local if each $M_{n}$ is $T$-local. 
By results of Waner [16], each $X_{n}$ has the homotopy type of a $G$-CW complex. However, as in the nonequivariant case, this property is lost upon passage to inverse limits and $G$-towers are generally not of the homotopy type of $G-C W$ complexes. This motivates our earlier discussion of $G-\mathrm{CW}$ approximation.

Proposition 7. Let $Z$ be a T-local G-tower. If $f: X \rightarrow Y$ is a T-cohomology isomorphism, then

is a bijection.

$$
f^{*}:[Y, Z]_{G} \rightarrow[X, Z]_{G}
$$

Proof. We may assume that $X$ and $Y$ are $G$-CW complexes, so that both the hypothesis and the conclusion are concerned with homotopy classes of $G$-maps. The proof is then an induction over "cocells" word for word dual to the good proof of Proposition 1 in [16]. Details of such arguments by duality will appear in [11], from which source the present pattern of development is taken.

The previous result is too general to be useful because it is too hard to recognize when a $G$-space is weakly $G$-equivalent to a $G$-tower. It is for this reason that the refined notions of simple and nilpotent $G$-towers were introduced.

Proposition 8. Let $X$ be a G-CW complex.

(i) If $X$ is $G$-simple, there exist a simple G-tower $Y$ and a weak G-equivalence $X \rightarrow Y$.

(ii) If $X$ is G-nilpotent, there exist a nilpotent G-tower $Y$ and a weak G-equivalence $X \rightarrow Y$.

(iii) If $X$ is $G$-nilpotent and each $X^{H}$ is $T$-local, there exist a $T$-local nilpotent $G$-tower $Y$ and a weak $G$-equivalence $X \rightarrow Y$.

Proof. The argument is precisely analogous to the nonequivariant construction of Postnikov systems (e.g. $[1,5,11]$ ). For finite $G$ (and $G$-simply connected $X$ ) complete details of (i) are in [13]. For general $G$, the argument is sketched in [4]. There is one point at which equivariance plays a substantive role. In (ii) and (iii), we are given that each $\pi_{1}\left(X^{H}\right)$ is nilpotent and acts nilpotently on $\pi_{n}\left(X^{H}\right)$ for $n \geqslant 2$. To construct $Y$, we need to know that the functor $\pi_{1}(X)$ from $\mathcal{O}$ to groups is nilpotent and that this functor acts nilpotently on the functor $\pi_{n}(X): \mathcal{O} \rightarrow \mathrm{Ab}$ for $n \geqslant 2$. These assertions hold by the functoriality of the relevant lower central series. For a group $\pi, \Gamma^{1} \pi=\pi$ and $\Gamma^{k+1} \pi=\left[\Gamma^{k} \pi, \pi\right]$. For a $\pi$-module $A, \Gamma^{1} A=A$ and $\Gamma^{k+1} A$ is the subgroup generated by $\left\{x a-a \mid x \in \pi\right.$ and $\left.a \in \Gamma^{k} A\right\}$. By functoriality, we obtain a descending sequence $\left\{\Gamma^{k} \pi_{n}(X)\right\}$ of sub-functors of $\pi_{n}(X)$ for $n \geqslant 1$, and $\Gamma^{k} \pi_{n}(X)$ is the trivial functor for $k$ sufficiently large by our definition of $G$-nilpotency. We construct $Y$ from the Eilenberg-MacLane $G$-spaces $K\left(\Gamma^{k} \pi_{n}(X) / \Gamma^{k+1} \pi_{n}(X), n\right)$. If each $X^{H}$ is $T$-local, then each $\Gamma^{k} \pi_{n}\left(X^{H}\right)$ and each quotient $\Gamma^{k} \pi_{n}\left(X^{H}\right) / \Gamma^{k+1} \pi_{n}\left(X^{H}\right)$ is $T$-local [5, I.2.7], hence $Y$ is $T$-local. We remark that the details can be carried out purely homotopically, without use of algebraic devices such as the universal coefficients theorem.

We put things together to obtain the following results. 
THEOREM 9. Let $Z$ be a G-nilpotent $G$-space. Then the following conditions are equivalent. When they hold, $Z$ is said to be a $T$-local $G$-space.

(i) Each $Z^{H}$ is $T$-local in the nonequivariant sense.

(ii) If $f: X \rightarrow Y$ is a T-cohomology isomorphism of $G$-spaces, then

is a bijection.

$$
f^{*}:[Y, Z]_{G} \rightarrow[X, Z]_{G}
$$

Proof. If (i) holds, then $Z$ is weakly $G$-equivalent to a $T$-local nilpotent $G$-tower by Proposition 8 and therefore (ii) holds by Proposition 7. Assume (ii). If $f: X \rightarrow Y$ is a nonequivariant $T$-cohomology isomorphism, then application of (ii) to the $T$-cohomology isomorphism

$$
1 \wedge f:(G / H)_{+} \wedge X \rightarrow(G / H)_{+} \wedge Y
$$

and use of the natural isomorphisms above Lemma 5 yield that

$$
f^{*}:\left[Y, Z^{H}\right] \rightarrow\left[X, Z^{H}\right]
$$

is a bijection. This verifies that the nilpotent space $Z^{H}$ satisfies what ought to be considered the defining property of a $T$-local space [11]; although precisely this criterion seems not to be stated elsewhere in the literature, it is implicit in [1, V.3.3].

The following is our main result.

THEOREM 10. Let $X$ be a G-nilpotent $G$-space. Then the following conditions on $a$ $G$-map $\lambda: X \rightarrow X_{T}$ from $X$ to a $T$-local $G$-space $X_{T}$ are equivalent.

(i) $\lambda^{*}:\left[X_{T}, Z\right]_{G} \rightarrow[X, Z]_{G}$ is a bijection for all $T$-local $G$-spaces $Z$.

(ii) $\lambda: X \rightarrow X_{T}$ is a $T$-cohomology isomorphism.

(iii) Each $\lambda^{H}: X^{H} \rightarrow X_{T}^{H}$ is a localization at $T$ in the nonequivariant sense.

There exists one and, up to isomorphism in HGT, only one G-map $\lambda$ satisfying these conditions; it is called the localization of $X$ at $T$.

Proof. Taking $Z=K(M, n)$ for $T$-local coefficient systems $M$, we see that (i) trivially implies (ii). By the previous theorem, (ii) implies (i). By Proposition 4, (iii) implies (ii). The uniqueness clause of the last statement will be immediate from (i). We shall construct $\lambda$ which satisfies (iii), hence also (i) and (ii). By uniqueness, this will automatically yield that (i) implies (iii) and so complete the proof.

As in the nonequivariant case $[5,12]$, there is a cellular construction which only works in the simply connected case and a cocellular construction which works in the nilpotent case. When $X$ is $G$-simply connected, we may assume without loss of generality that $X$ is a $G-C W$ complex with a single vertex, no 1-cells, and based attaching maps. The last condition implies that the domains of attaching maps are $G$-spaces of the form $(G / H)_{+} \wedge S^{n}$. Let $\lambda: S^{n} \rightarrow S_{T}^{n}$ be a localization of $S^{n}$ at $T$ and continue to write $\lambda$ for its extensions

$$
1 \wedge \lambda:(G / H)_{+} \wedge S^{n} \rightarrow(G / H)_{+} \wedge S_{T}^{n}
$$


At least for $n \geqslant 2$ (but not for $n=1$, where these spaces need not be nilpotent), these $G$-maps are localizations at $T$ by Lemma 5 and the observation that

$$
\left[(G / H)_{+} \wedge X\right]^{K}=(G / H)_{+}^{K} \wedge X
$$

for $G$-trivial $X$ and thus $(G / H)_{+} \wedge S_{T}^{n}$ is $T$-local. The following construction completes the proof of the theorem in the $G$-simply connected case.

Construction 11 . Let $X$ be a $G$-simply connected $G$-CW complex, as above. We construct compatible localizations $\lambda_{n}: X^{n} \rightarrow X_{T}^{n}$ of the skeleta of $X$. We proceed by induction on $n$, starting with the observation that $X^{2}$ is a wedge of $G$-spaces of the form $(G / H)_{+} \wedge S^{2}$. Let $X^{n+1}$ be the cofibre of $j_{n}: J_{n} \rightarrow X^{n}$, where $J_{n}$ is a wedge of $G$-spaces of the form $(G / H)_{+} \wedge S^{n}$, and assume that we have constructed $\lambda_{n}$. Consider the following diagram, where $\lambda: J_{n} \rightarrow\left(J_{n}\right)_{T}$ is the obvious wedge of localizations:

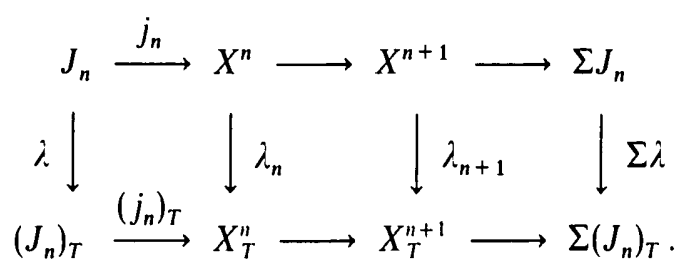

Since $X_{T}^{\prime \prime}$ is $T$-local, there exists $\left(j_{n}\right)_{T}$ such that the left square is $G$-homotopy commutative. Let $X_{T}^{n+1}$ be the cofibre of $\left(j_{n}\right)_{T}$. Then the rows are cofibre sequences. The standard Barratt-Puppe sequence construction gives a $G$-map $\lambda_{n+1}$ such that the right square is $G$-homotopy commutative and the middle square is actually commutative. Since passage to fixed point spaces preserves cofibre sequences, $X_{T}^{n+1}$ is $T$-local, each $\lambda_{n+1}^{H}$ is a localization at $T$, and therefore $\lambda_{n+1}$ is a localization at $T$. On passage to unions, we obtain the desired localization $\lambda: X \rightarrow X_{T}$.

Using Elmendorf's functorial construction of spaces $K(M, n)$, we see that the localization $M \rightarrow M_{T}$ of a coefficient system $M$ induces a $G$-map

$$
\lambda: K(M, n) \rightarrow K\left(M_{T}, n\right),
$$

and $\lambda$ is a localization at $T$ since its restrictions

$$
i^{H}: K(M, n)^{H}=K(M(G / H), n) \rightarrow K\left(M(G / H)_{T}, n\right)=K\left(M_{T}, n\right)^{H}
$$

are localizations at $T$. To complete the proof of Theorem 10, we may assume that our $G$-nilpotent $G$-space $X$ is given as a nilpotent $G$-tower. We adopt the notations of Definition 6 and have the following dual to the previous construction.

Construction 12. Let $X$ be a nilpotent $G$-tower. We construct compatible localizations $\lambda_{n}: X_{n} \rightarrow\left(X_{n}\right)_{T}$ by induction on $n$, starting with $\left(X_{0}\right)_{T}=\{*\}$. Consider the following diagram, where the top fibration and the localization $\lambda_{n}$ are given and where $i$ is the localization just constructed:

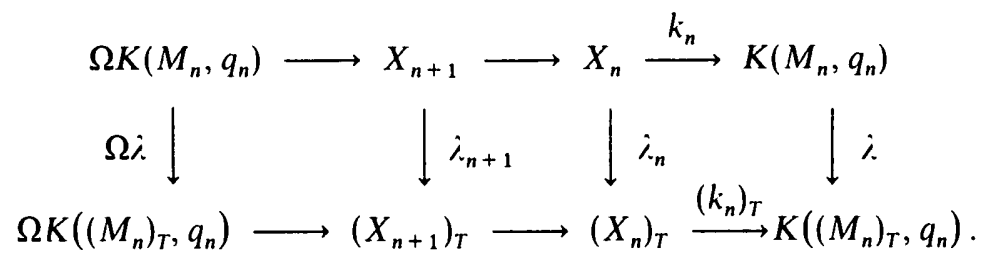


By the universal property of $\lambda_{n}$, there exists $\left(k_{n}\right)_{T}$ such that the right square is $G$-homotopy commutative. Let $\left(X_{n+1}\right)_{T}$ be the fibre of $\left(k_{n}\right)_{T}$. Again, the standard Barratt-Puppe sequence construction gives a $G$-map $\lambda_{n+1}$ such that the left square is $G$-homotopy commutative and the middle square is actually commutative. Since passage to fixed point spaces preserves fibre sequences, $\left(X_{n+1}\right)_{T}$ is $T$-local, each $\lambda_{n+1}^{H}$ is a localization at $T$, and therefore $\lambda_{n+1}$ is a localization at $T$. On passage to limits, we obtain the desired localization $\lambda: X \rightarrow X_{T}$.

Remark 13. For finite $G$, the semi-simplicial construction of Bousfield and Kan [1] automatically generalizes to a functorial (but not continuously functorial) construction of equivariant localizations. Of course, this fails for general compact Lie groups $G$.

Remark 14. For finite $G$, the equivariant analog of Sullivan's algebraization of rational homotopy theory has been developed in [14]. Rational Hopf $G$-spaces have been studied in [15]; they split as products of spaces $K(M, n)$ when $G=Z_{p} i$ but they need not so split when $G=Z_{p} \times Z_{q}$.

We have left one unfinished piece of business.

Proof of Proposition 2. Suppose the conclusion is false. Since there are no infinite descending chains of compact Lie groups, we can choose a minimal subgroup $H$ for which the conclusion fails. Starting over with $G$ replaced by $H$, we may assume that $f: X \rightarrow Y$ is a $G$-map such that $f_{*}: E_{*}(X / H) \rightarrow E_{*}(Y / H)$ is an isomorphism for all $H$ in the family $F$ of proper (closed) subgroups of $G$ and $f_{*}: E_{*}\left(X^{H}\right) \rightarrow E_{*}\left(Y^{H}\right)$ is an isomorphism for all $H$ in the family $F_{\infty}$ of all (closed) subgroups of $G$. Write $E_{*}^{G}(X)=E_{*}(X / G)$. Then $E_{*}^{G}$ is a $Z$-graded $G$-homology theory. As explained by tom Dieck [3, §7.3], one can "localize" equivariant homology theories at families of subgroups or between pairs of families. Thus one has localized theories $E_{*}^{G}[F]$ and $E_{*}^{G}\left[F_{\infty}, F\right]$. These fit into a natural long exact sequence, and we have a commutative diagram with exact rows

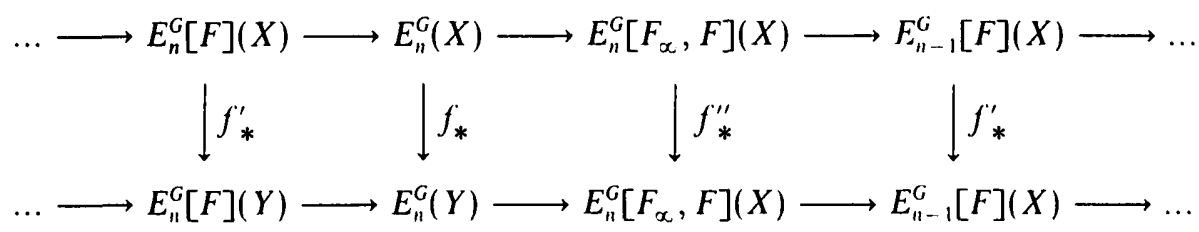

all vertical arrows being induced by $f$. We shall prove that $f_{*}^{\prime}$ and $f_{*}^{\prime \prime}$ are isomorphisms and so conclude that $f_{*}$ is an isomorphism. Since $(G / H \times X) / G \cong X / H$, our first hypothesis asserts that

$$
(1 \times f)_{*}: E_{*}^{G}(G / H \times X) \rightarrow E_{*}^{G}(G / H \times Y)
$$

is an isomorphism for all $H \in F$. By the homology version of Jackowski [7, 1.4], we see at once that $f_{*}^{\prime \prime}$ is an isomorphism. By tom Dieck $[3,7.4 .2]$, the natural map

$$
E_{*}^{G}\left[F_{\infty}, F\right]\left(X^{G}\right) \rightarrow E_{*}^{G}\left[F_{\infty}, F\right](X)
$$


is an isomorphism, and similarly for $Y$. Consider the diagram above with $X$ and $Y$ replaced by $X^{G}$ and $Y^{G}$. Here $f_{*}^{\prime}$ is again an isomorphism, since $\left(G / H \times X^{G}\right) / G \cong X^{G}$, but now $f_{*}$ is also an isomorphism since $E_{*}^{G}\left(X^{G}\right)=E_{*}\left(X^{G}\right)$. Thus $f_{*}^{\prime \prime}$ is an isomorphism in the new diagram, hence also in the old diagram, and the proof is complete. Note that we only used our second hypothesis on $X^{G}$ and not on $X^{H}$ for proper subgroups $H$. (New proofs of the results quoted in this argument will appear in [9], which will give a systematic account of equivariant homology theory.)

\section{References}

1. A. K. Bousfield and D. M. KAN, Homotopy limits, completions, and localizations, Lecture Notes in Mathematics 304 (Springer, Berlin, 1972).

2. G. BREDON, Equitariant cohomology theories, Lecture Notes in Mathematics 34 (Springer, Berlin, 1967).

3. T. TOM DIECK, Transformation groups and representation theory, Lecture Notes in Mathematics 766 (Springer, Berlin, 1979).

4. A. Elmendorf, 'Equivariant homotopy and fixed point sets', Trans. Amer. Math. Soc., to appear.

5. P. Hilton, G. Mislin and J. RoitberG, Localization of nilpotent groups and spaces, Mathematics Studies 15 (North-Holland Publishing Co., 1975).

6. S. Illman, 'Equivariant singular homology and cohomology I', Mem. Amer. Math. Soc., 156, 1975.

7. S. Jackowski, Equicariant K-theory and cyclic subgroups, London Math. Soc. Lecture Note Series 26, 1977, pp. 76-92.

8. G. Lewis, J. P. MAY and J. MCClure, 'Ordinary RO(G)-graded cohomology', Bull. Amer. Math. Soc., 4 (1981), 208-212.

9a. G. Lewis, J. P. May, J. MCClure and M.Steinberger, 'Equivariant stable homotopy theory', Springer Lecture Notes in Mathematics, in preparation.

9b. G. LeWIS, J. P. MAY, J. MCClure and S. WANER, 'Equivariant cohomology theory', in preparation.

10. T. Matumoto, 'On G-CW-complexes and a theorem of J. H. C. Whitehead', J. Fac. Sc. Unic. Tokyo Sect. IA Math., 18 (1971/72), 363-374.

11. J. P. MAY, The homotopical foundations of algebraic topology (Academic Press), in preparation.

12. D. Sullivan, 'The genetics of homotopy theory and the Adams conjecture', Ann. of Math., (2), 100 (1974), 1-79.

13. G. Triantafillou, Äquicariante rationale Homotopietheorie (Bonner Math. Schriften Nr. 110, 1978).

14. G. Triantafillou, 'Equivariant minimal models', Trans. Amer. Math. Soc., to appear.

15. G. Triantafillou, 'Rationalization of Hopf $G$-spaces', preprint.

16. S. WANER. 'Equivariant homotopy theory and Milnor's theorem', Trans. Amer. Math. Soc., 258 (1980), $351-368$.

17. S. J. Willson, 'Equivariant homology theories on G-complexes', Trans. Amer. Math. Soc., 212 (1975), $155-171$.

Department of Mathematics, University of Chicago, Chicago,

Illinois 60637 , U.S.A.

School of Mathematics,

University of Minnesota,

Minneapolis,

Minnesota 55455, U.S.A.
Department of Mathematics, Johns Hopkins University, Baltimore, Maryland 21218, U.S.A. 